7th International Workshop on Astronomy and

Relativistic Astrophysics (IWARA 2016)

International Journal of Modern Physics: Conference Series

Vol. 45 (2017) 1760065 (7 pages)

(C) The Author(s)

DOI: $10.1142 / \mathrm{S} 2010194517600655$

\title{
Double Heavy Quark-Antiquark Pair Production in Double Parton Scattering Processes at the LHC
}

\author{
Cristiano B. Mariotto and Rafael P. da Silva \\ Instituto de Matemática, Estatística e Física \\ Universidade Federal do Rio Grande (FURG) \\ Av. Itália, $\mathrm{km} \mathrm{8,} \mathrm{Bairro} \mathrm{Carreiros}$ \\ 96203-900 Rio Grande, Rio Grande do Sul, Brazil \\ cristianomariotto@furg.br,palota.rafael@gmail.com
}

Published 15 August 2017

\begin{abstract}
The high gluon density in the initial state of hadronic collisions at LHC energies implies that the probability of multiple parton interactions within one proton-proton collision increases. In particular, this motivates one to investigate the importance of Double Parton Scattering (DPS) processes at the LHC. In a DPS process one can have, from one proton-proton collision, two interacting partons coming from each colliding hadron. In this context, the relation between the double parton distribution functions (dPDF) and the usual parton distribution functions (PDF) is discussed, and some formulae for the DPS cross section are compared. Our results focus on the production of different final states related to two heavy quark-antiquark pairs. Those include $c \bar{c} c \bar{c}$, which is called the golden channel of DPS, $b \bar{b} b \bar{b}$ and $c \bar{c} b \bar{b}$.
\end{abstract}

Keywords: DPS, QCD, Heavy Quarks.

PACS numbers: 13.85.-t, 11.80.La, 14.65.Dw, 14.65.Fy

\section{Introduction}

Although multiple particle interactions in a single proton-proton collisions are studied for quite some time, they became relevant only with the advent of the LHC, because of the high parton luminosity of the high energy proton beams in initial state. Since then, there are lots of activities in the area of double parton scattering processes, both theoretically ${ }^{1-8}$ and experimentally ${ }^{9-13}$.

Most processes are studied in the framework of the Single Parton Scattering processes (SPS), where one has the usual QCD factorization formula written for

This is an Open Access article published by World Scientific Publishing Company. It is distributed under the terms of the Creative Commons Attribution 4.0 (CC-BY) License. Further distribution of this work is permitted, provided the original work is properly cited. 


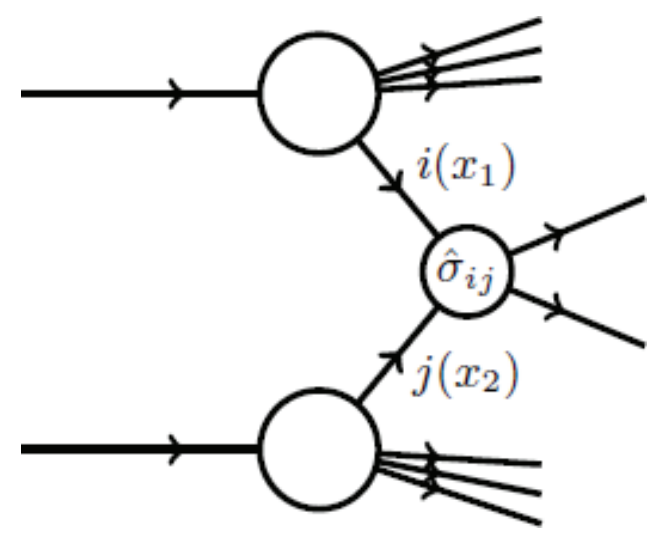

Fig. 1. A general SPS process.

hadron-hadron collisions 14

$$
\sigma^{S P S}=\sum_{i, j} \int d x_{1} d x_{2} f_{i}^{A}\left(x_{1}, \mu\right) f_{j}^{B}\left(x_{2}, \mu\right) \hat{\sigma}_{i j}\left(x_{1} x_{2} \sqrt{s}, \mu\right)
$$

where $f_{i}^{A}\left(x_{1}, \mu\right)$ and $f_{j}^{B}\left(x_{2}, \mu\right)$ are the parton distribution functions (nonperturbative), and $\hat{\sigma}_{i j}$ are the partonic cross sections (perturbative) for the contributing sub-processes. Here two partons (one from each hadron) interact, producing two or more partons in the hard scattering.

In a Double Parton Scattering (DPS) process one can have, from one protonproton collision, two interacting partons coming from each colliding hadron, as

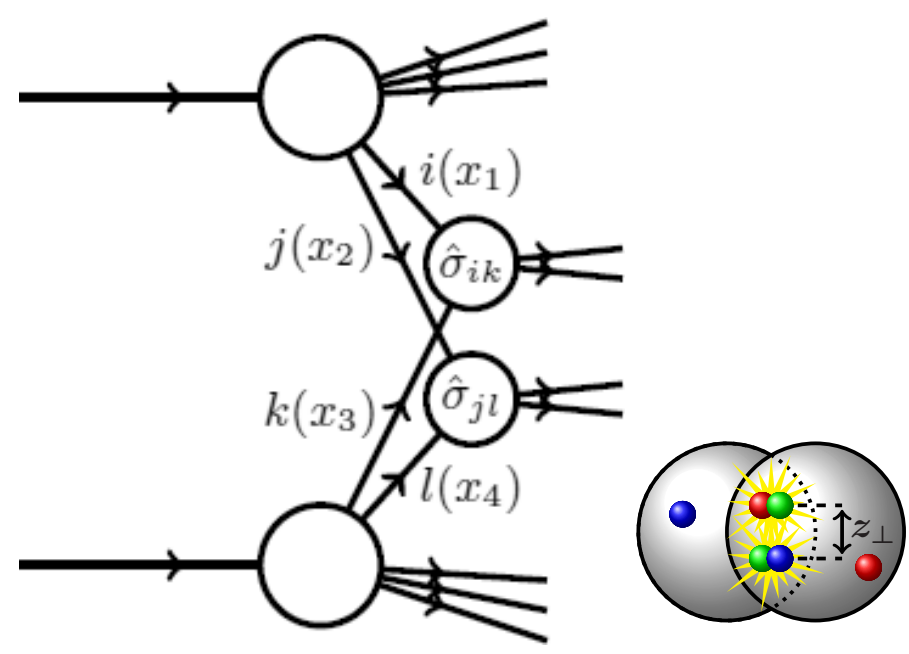

Fig. 2. Representation of a general DPS process (left) and its view from the transverse plane (right). 
shown in Fig. 2. The DPS cross section can be written as ${ }^{3}$

$$
\begin{array}{r}
d \sigma=\frac{1}{S} \sum_{i, j, k, l} \int d^{2} \boldsymbol{z}_{\perp} F_{i j}\left(x_{1}, x_{2}, \boldsymbol{z}_{\perp}, \mu\right) F_{k l}\left(x_{3}, x_{4}, \boldsymbol{z}_{\perp}, \mu\right) \\
\hat{\sigma}_{i k}\left(x_{1} x_{3} \sqrt{s}, \mu\right) \hat{\sigma}_{j l}\left(x_{2} x_{4} \sqrt{s}, \mu\right),
\end{array}
$$

where $F_{i j}$ and $F_{k l}$ are the double parton distribution functions (dPDF), with two partons coming from each proton, and $\hat{\sigma}_{i k}$ and $\hat{\sigma}_{j l}$ are the partonic cross sections. Also, $S$ is a symmetry factor, being (2) 1 for (in)distinguishable final states, $\boldsymbol{z}_{\perp}$ is the transverse separation between the two partonic interactions, $x_{i}(i=1,2,3$ and 4 ) is the proton momentum fraction carried by the corresponding parton. The dPDF's in the general form above, including the $\boldsymbol{z}_{\perp}$ dependency are unknown at present. If one assumes that the transverse separation $z_{\perp}$ is not correlated with the longitudinal momentum fractions, one could write

$$
F_{i j}\left(x_{1}, x_{2}, z_{\perp}, \mu\right)=F_{i j}\left(x_{1}, x_{2}, \mu\right) G\left(z_{\perp}, \mu\right) .
$$

Integrating Eq. (2) over the transverse separation, one obtains

$$
d \sigma=\frac{1}{S \sigma_{e f f}} \sum_{i, j, k, l} F_{i j}\left(x_{1}, x_{2}, \mu\right) F_{k l}\left(x_{3}, x_{4}, \mu\right) \hat{\sigma}_{i k}\left(x_{1} x_{3} \sqrt{s}, \mu\right) \hat{\sigma}_{j l}\left(x_{2} x_{4} \sqrt{s}, \mu\right),
$$

where one can define the effective cross section

$$
\sigma_{e f f}=\left[\int d^{2} \boldsymbol{z}_{\perp} G\left(\boldsymbol{z}_{\perp}, \mu\right)^{2}\right]^{-1}
$$

which is a measure of the proton area and should be determined from experiments ${ }^{11}$. In this work we have adopted, as in Ref. $4, \sigma_{\text {eff }}=15 \mathrm{mb}$.

The somewhat less general $F_{i j}\left(x_{1}, x_{2}, \mu\right)$ has the physical meaning of the probability of having two partons, $i$ and $j$, carrying the momentum fraction $x_{1}$ and $x_{2}$ of the same proton (a similar interpretation applies for $F_{k l}\left(x_{3}, x_{4}, \mu\right)$ ). The dPDF's obey the double DGLAP (dDGLAP) evolution equations ${ }^{1}$ and a couple of sum rules ${ }^{7}$, in an analogous way as the usual PDF's. For example, the dDGLAP evolution equation for the two-gluon $\mathrm{dPDF}$ can be written as

$$
\mu \frac{d}{d \mu} F_{g g}\left(x_{1}, x_{2}, z_{\perp}, \mu\right)=\frac{\alpha_{s} C_{F}}{\pi}\left\{\sum _ { i } \left[\int \frac{d y}{y} P_{g i}\left(\frac{x_{1}}{y}\right) F_{i g}\left(y, x_{2}, \boldsymbol{z}_{\perp}, \mu\right)\right.\right.
$$

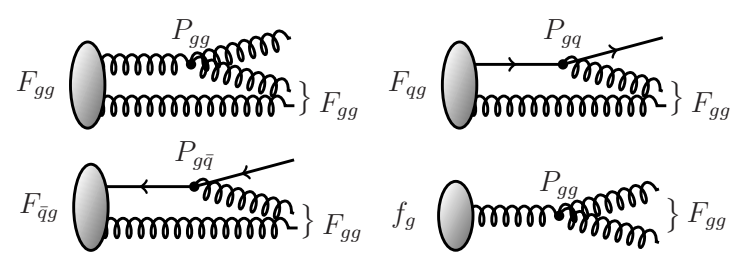

Fig. 3. Representation of some contributing diagrams for the dDGLAP evolution equation for the gluon-gluon dPDF $F_{g g}$. 


$$
\left.\left.+\int \frac{d y}{y} P_{g i}\left(\frac{x_{2}}{y}\right) F_{g i}\left(x_{1}, y, \boldsymbol{z}_{\perp}, \mu\right)\right]+P_{g g}\left(\frac{x_{2}}{y}\right) \frac{f_{g}\left(x_{1}+x_{2}, \mu\right)}{x_{1}+x_{2}}\right\},
$$

where $P_{g i}, i=q, \bar{q}, g$ are the usual splitting functions ${ }^{14}$, and is represented in Fig. 3. As shown, the gluon-gluon double distribution in one single proton can be originated from a gluon-gluon, a quark-gluon or an antiquark-gluon pair. An anomalous contribution is represented in the last graph (last term in Eq. (6)), where a gluon-gluon pair is originated from a single gluon, in this last term one has a connection between the gluon PDF and the gluon-gluon $\mathrm{dPDF}$.

\subsection{DPS: a simplified approach}

The double parton distributions can be further simplified with the assumption in Eq. (3) and the Ansatz

$$
F_{i j}\left(x_{1}, x_{2}, \mu\right)=f_{i}\left(x_{1}, \mu\right) f_{j}\left(x_{2}, \mu\right) \theta\left(1-x_{1}-x_{2}\right)\left(1-x_{1}-x_{2}\right),
$$

where $f_{i}$ and $f_{j}$ are the usual parton distribution functions (PDF's). Due to the last two terms at small- $x$ the above expression becomes

$$
F_{i j}\left(x_{1}, x_{2}, \mu\right)=f_{i}\left(x_{1}, \mu\right) f_{j}\left(x_{2}, \mu\right) .
$$

Replacing Eq.(8) in Eq.(4) and integrating over the momentum fractions, one obtains

$$
\sigma=\sum_{i, j, k, l} \frac{\sigma_{i k} \sigma_{j l}}{S \sigma_{e f f}}
$$

which is the so called "pocket formula" for DPS processes.

\section{Double Heavy Quark-Antiquark Pair Production}

In this section we present our results for heavy quark-antiquark pair production at the LHC via Double Parton Scattering processes. Although the charm quark production $(c \bar{c} c \bar{c})$ is known to be the golden channel and has been more extensively studied, we are also interested in final states containing $b$ quarks.

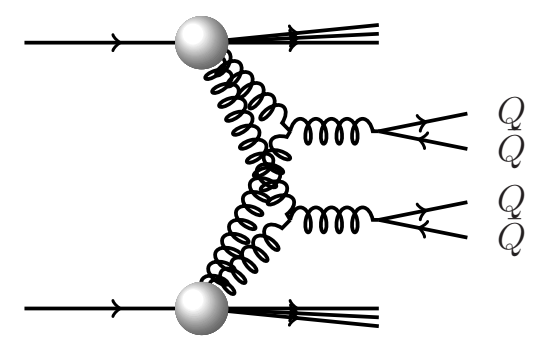

Fig. 4. Representation of a typical DPS process leading to a $Q \bar{Q} Q \bar{Q}$ in the final state. 
A typical contributing diagram to $Q \bar{Q} Q \bar{Q}$ produced in DPS is shown in Fig. 4. The differential cross section can be written in terms of the pocket formula as

$$
\frac{d \sigma^{D P S}}{d y_{1} d y_{2} d^{2} p_{T_{A}} d y_{3} d y_{4} d^{2} p_{T_{B}}}=\frac{1}{S \sigma_{e f f}} \frac{d \sigma_{i k}}{d y_{1} d y_{2} d^{2} p_{T_{A}}} \frac{d \sigma_{j l}}{d y_{3} d y_{4} d^{2} p_{T_{B}}}
$$

where the subscripts A and B refer to the first and second parton scattering, respectively. For the first SPS process, one has ${ }^{14}$

$$
\frac{d \sigma}{d y_{1} d y_{2} d^{2} p_{T}}=\frac{1}{64 \pi^{2} m_{T}^{4}(1+\cosh \Delta y)^{2}} \sum_{i k} x_{1} f_{i}\left(x_{1}, \mu^{2}\right) x_{2} f_{k}\left(x_{2}, \mu^{2}\right) \bar{\sum}|\mathcal{M}|^{2}
$$

where $m_{T}=\sqrt{m^{2}+p_{T}^{2}}$ is the transverse mass of a heavy $Q(\bar{Q}), \Delta y=y_{1}-y_{2}$ is the rapidity difference between the $Q$ and $\bar{Q}$ from a given SPS process. The momentum fractions of the initial partons can be written in terms of those rapidities as $x_{1}=$ $\frac{m_{T}}{\sqrt{s}}\left(e^{y_{1}}+e^{y_{2}}\right)$ and $x_{2}=\frac{m_{T}}{\sqrt{s}}\left(e^{-y_{1}}+e^{-y_{2}}\right)$. The expression for the second scattering is the same as above with the exchanges of $1 \rightarrow 3$ and $2 \rightarrow 4$. The contributing sub-processes in leading order (LO) accuracy are $g g \rightarrow Q \bar{Q}$ and $q \bar{q} \rightarrow Q \bar{Q}$, but the gluon initiated processes dominate at LHC energies. In our results using the pocket formula we use the CTEQ6L ${ }^{15}$ for the parton distributions $f_{i}$ and $f_{k}$.

From here on, we show a few results for the production of $c \bar{c} c \bar{c}$, and $b \bar{b} b \bar{b}$ in DPS processes, starting from Eq. (10) and integrating over the appropriate variables. To obtain the rapidity distribution of one heavy quark, one integrates over the transverse momenta and rapidities of the other three partons. The results are shown in Fig. 5. The typical magnitude of the cross sections indicate the feasibility of the final states. The uncertainties in these predictions are investigated by varying the charm mass, $1.3 \mathrm{GeV}<m_{c}<1.5 \mathrm{GeV}$, and the factorization scale, $Q=\mu_{F}=M_{c \bar{c}}, m_{T}$, meaning the invariant mass of a $c \bar{c}$ pair and the transverse mass of the considered
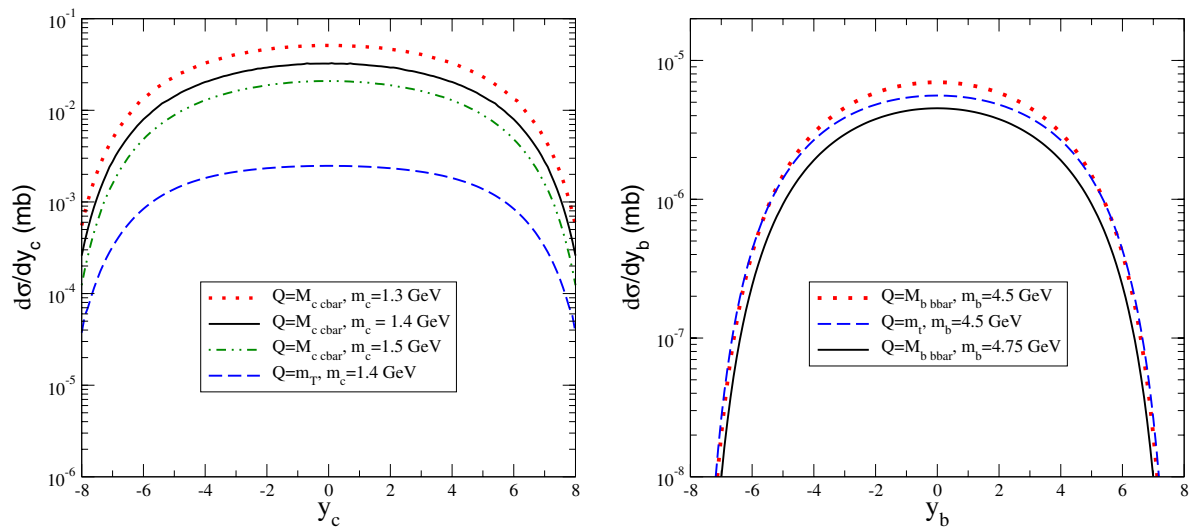

Fig. 5. Rapidity distribution of the $c$ and $b$ quarks from the final states $c \bar{c} c \bar{c}$ (left) and $b \bar{b} b \bar{b}$ (right) produced in DPS processes. Uncertainties in the quark mass and in the factorization scale are also shown. 
charm quark. The mass and scale uncertainties are amplified because we are here using two LO cross sections. For the $b \bar{b} b \bar{b}$ final state, the smaller cross sections are still large enough to be feasible. The bottom quark mass is taken as $4.5 \mathrm{GeV}<m_{b}<$ $4.75 \mathrm{GeV}$, and the factorization scale is $Q=\mu_{F}=M_{b \bar{b}}, m_{T}$. Compared with the previous case one has a somewhat lower mass and scale uncertainties.

In order to get more accurate results, one could go to NLO accuracy or use the $k_{T}$ factorization approach ${ }^{5}$, which go beyond the LO results of the collinear approach.
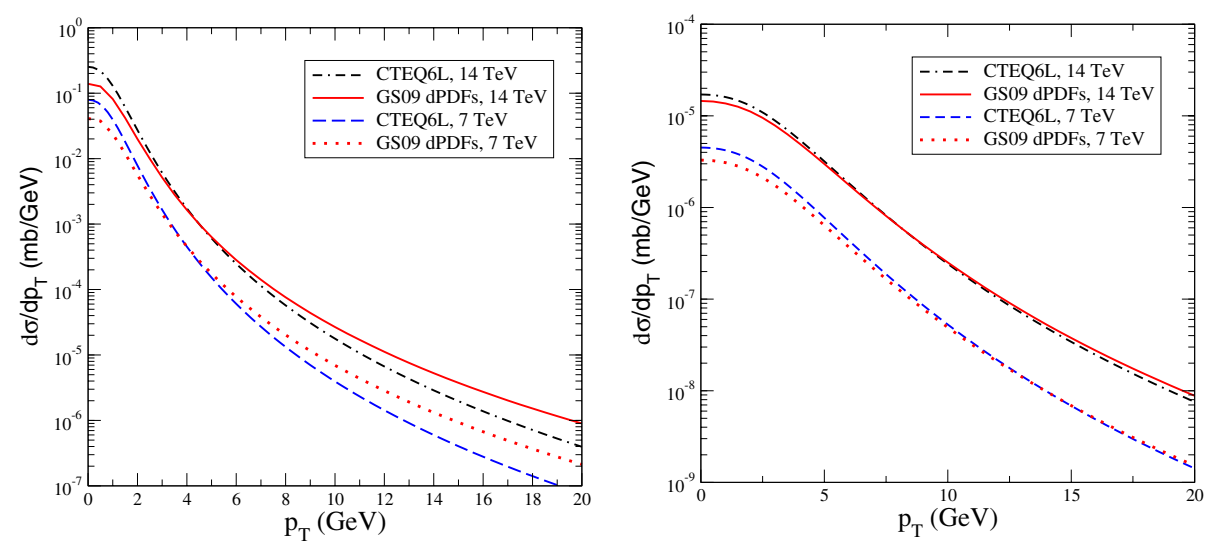

Fig. 6. Transverse momentum distribution of the $c(b)$ quark from the final states $c \bar{c} c \bar{c}$ (left) and $b \bar{b} b \bar{b}$ (right) produced in DPS processes, comparing the pocket formula and and considering dPDF's.

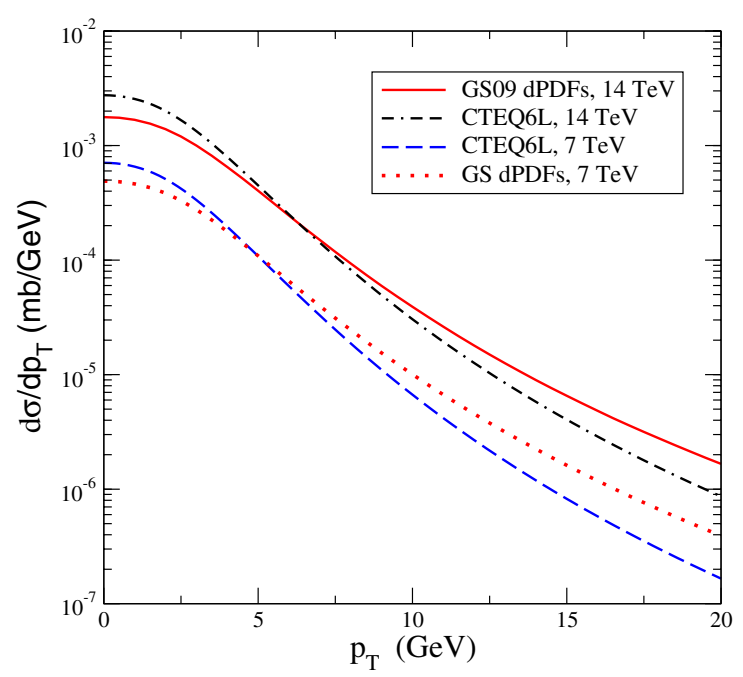

Fig. 7. Transverse momentum distribution of the $c$ quark from the final state $c \bar{c} b \bar{b}$ produced in DPS processes, comparing the pocket formula and and considering dPDF's. 
Another source of uncertainty is the very use of the pocket formula, which is certainly not exact and neglects correlations between the partons coming from a single proton. In what follows we try to go beyond it, considering the double PDF's available in the literature ${ }^{1}$ and the intermediate expression, Eq. (4). In Fig. 6 we show our results for the transverse momentum distributions for the production of $c \bar{c} c \bar{c}$ (left panel) and $b \bar{b} b \bar{b}$ (right panel) in DPS processes, comparing the pocket formula and its improvement considering the dPDF's. We can observe a slower $p_{T}$ decrease for dPDF's than for the pocket formula. In Fig. 7 we show the corresponding results for the production of the mixed state $c \bar{c} b \bar{b}$. The same behaviour is observed, and the cross section values are between the pure charm and pure bottom case.

\section{Conclusions}

Double parton scattering processes have been studied and measured at the LHC. The most commonly used theoretical approach is to consider the pocket formula, in which one writes the DPS cross section from a combination of two SPS cross sections, besides a combinatorial factor and an effective cross section. However, the more appropriate quantities to consider in the initial state are the double parton distributions, which are non-perturbative quantities obeying a pQCD evolution equation.

In this contribution we present some results for heavy quark-antiquark pair production at the LHC via DPS processes, using the so called pocket formula and its improvement. Although charm production is considered to be the golden channel, final states containing $b \bar{b}$ pairs are also feasible. The next steps include to keep going beyond the pocket formula, and considering correlations among different final state particles.

\section{References}

1. J. R. Gaunt and W. J. Stirling, JHEP 03, 005 (2010).

2. M. Diehl and A. Shafer, Phys. Lett. B 698, 389-402 (2011).

3. A. V. Manohar and W. J. Waalewijn, Phys. Rev. D 85, 114009 (2012).

4. M. Luszczak, R. Maciula, and A. Szczurek, Phys. Rev. D 85, 094034 (2012).

5. R. Maciula and A. Szczurek, Phys. Rev. D 87, 074039 (2013).

6. F. A. Ceccopieri, Phys. Lett. B 734, 79-85 (2014).

7. K. Golec-Biernat and E. Lewandowska, Phys. Rev. D 90, 014032 (2014).

8. K. Kutak, R. Maciula, M. Serino, A. Szczurek, and A. van Hameren JHEP 04, 175 (2016).

9. R. Aij et al., JHEP 06, 141 (2012).

10. G. Aad et al., New J. Phys. 15, 033038 (2013).

11. S. Chatrchyan et al., Phys. Rev. D 89, 092010 (2014).

12. G. Aad et al., JHEP 12, 105 (2015).

13. V. M. Abazov et al., Phys. Rev. Lett. 116, 082002 (2016).

14. R. K. Ellis, W. J. Stirling, and B. R. Webber, QCD and Collider Physics, (Cambridge University Press, Cambridge, 2003).

15. J. Pumplin, D. R. Stump, J. Huston, H. L. Lai, P. M. Nadolsky, and W. K. Tung, J. High Energy Phys. 07, 012 (2002). 